\title{
Réalisation industrielle de miroirs multicouches pour optique $\mathbf{X}$
}

P. Davi

MATRA DETIDTO, 14 rue Paul Dautier, 78140 Vélizy Villacoublay, France

A la suite d'un contrat d'études avec le CEA, le Laboratoire Couches Minces de MATRA est, maintenant, en mesure de fournir de façon industrielle des miroirs sélectifs multicouches, jusqu'à des longueurs d'onde d'utilisation aussi courtes que $1 \AA$ selon l'angle d'incidence. Répondant à la demande pour des besoins spécifiques, nous optimisons, de manière théorique, l'empilement par le choix des matériaux, le nombre de périodes et les épaisseurs des couches.

Le procédé de fabrication employé, par pulvérisation cathodique magnétron, permet de rendre le dépôt entièrement automatique et traiter en même temps une grande quantité de substrats, de dimensions jusqu'à $\varnothing 100 \mathrm{~mm}$ ou rectangulaires de longueur 250 $\mathrm{mm}$.

Notre moyen de contrôle goniométrique, entièrement informatisé, spécifiquement réalisé par MATRA par l'Institut d'Optique, travaille à $1,54 \AA ̊$ et permet de mesurer la période de l'empilement et l'indice moyen de la multicouche.

Les premières mesures de réflectivité absolue ont été réalisées sur le synchrotron SUPER-ACO du L.U.R.E. à Orsay, sur des bancs de mesures spécifiques développées par les centres CEA de Limeil Valenton et Bruyères le Chatel.

Nos résultats expérimentaux nous placent au niveau des performances de la concurrence et de continuels nouveaux développements doivent nous permettre d'élargir encore cette gamme de produits 